\title{
An approach to confirm the existence of a black hole
}

Junjin Huang ${ }^{1}$, Chanyuk Lam ${ }^{1}$, Wanquan Liu$^{2}$, Qiuyun Liu ${ }^{1, *}$

${ }^{1}$ School of Life Sciences, Sun Yat-Sen University, Guangzhou 510275, China.

${ }^{2}$ Earthquake Monitoring and Prediction Department, Emergency Management Bureau, Deyang 618000, Sichuan, China.

*Correspondence author. E-mail address: liuqiuyunsysu@163.com 


\begin{abstract}
Solar and lunar gravitational pulls prompt slower accelerations of large mass or faster accelerations of small mass on the Earth. Gravitation-triggered acceleration and deceleration is the cause of volcanoes, earthquakes, sunspots and starspots. Starspots in neighboring stars can thus be used as the indication for the existence of a black hole.
\end{abstract}


The existence of black holes has been widely debated since Einstein proposed it in 1915. Now it is possible to verify the existence of black holes through starspots.

Sunspots arise mostly due to the effect of Jupiter's increasing gravitational pull as Jupiter approaches perihelion. Solar and lunar pulls triggers slower accelerations of large mass or faster accelerations of small mass on the Earth (1-3), in a way similar to fast-traveling trains and cars on the same road with speed changes causing crashes. This principle of gravitational acceleration and deceleration applies to volcanoes and earthquakes as well as sunspots and starspots (1-4). Therefore, starspots in nearby stars can be adopted as the substantiation for the presence of a black hole (4).

\section{References}

1. Yan S, et al. Filtering out non-specific animal behavior to better predict $\begin{array}{llll}\text { impending } & \text { earthquakes. } & & \end{array}$ https://doi.org/10.31219/osf.io/bs3qd.

2. Yan S, et al. Earthquakes-an intricate trio dance of gravitation. Science 2018; https://www.science.org/doi/10.1126/science.aag0482.

3. Yan S, et al. Volcanoes or earthquakes: Wrist wrestling. Science, 2019; https://www.science.org/doi/10.1126/science.358.6370.1520.

4. Wang Y, et al. RE: "Direct imaging discovery of a Jovian exoplanet within a triple-star system".

Science, 2017; https://www.science.org/doi/10.1126/science.aaf9671. 